\title{
Maternal steroid levels and the autistic traits of the mother and infant
}

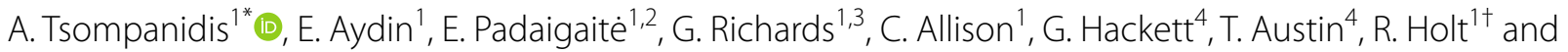 \\ S. Baron-Cohen ${ }^{1+}$
}

\begin{abstract}
Background: Prenatal sex steroids have been associated with autism in several clinical and epidemiological studies. It is unclear how this relates to the autistic traits of the mother and how early this can be detected during pregnancy and postnatal development.

Methods: Maternal serum was collected from pregnant women $(n=122)$ before or during their first ultrasound appointment [mean $=12.7(\mathrm{SD}=0.7)$ weeks]. Concentrations of the following were measured via immunoassays: testosterone, estradiol, dehydroepiandrosterone sulphate, progesterone; and sex hormone-binding globulin which was used to compute the free fractions of estradiol (FEI) and testosterone (FTI). Standardised human choriogonadotropin ( $\mathrm{hCG}$ ) and pregnancy-associated plasma protein A (PAPP-A) values were obtained from clinical records corresponding to the same serum samples. Mothers completed the Autism Spectrum Quotient (AQ) and for their infants, the Quantitative Checklist for Autism in Toddlers (Q-CHAT) when the infants were between 18 and 20 months old.
\end{abstract}

Results: FEl was positively associated with maternal autistic traits in univariate $(n=108$, Pearson's $r=0.22, p=0.019)$ and multiple regression models (semipartial $r=0.19, p=0.048$ ) controlling for maternal age and a diagnosis of PCOS. Maternal estradiol levels significantly interacted with fetal sex in predicting infant Q-CHAT scores, with a positive relationship in males but not females ( $n=100$, interaction term: semipartial $r=0.23, p=0.036$ ) after controlling for maternal AQ and other covariates. The opposite was found for standardised hCG values and Q-CHAT scores, with a positive association in females but not in males ( $n=151$, interaction term: $r=-0.25, p=0.005$ ).

Limitations: Sample size of this cohort was small, with potential ascertainment bias given elective recruitment. Clinical covariates were controlled in multiple regression models, but additional research is needed to confirm the statistically significant findings in larger cohorts.

Conclusion: Maternal steroid factors during pregnancy are associated with autistic traits in mothers and their infants.

Keywords: Autism, Autistic Traits, Prenatal, Pregnancy, Estradiol, Sex, Interaction

\section{Background}

Autism is a neurodevelopmental condition characterised by difficulties in social communication alongside unusually restricted interests, repetitive behaviour and frequent

\footnotetext{
*Correspondence: at768@medschl.cam.ac.uk

${ }^{\dagger} \mathrm{R}$. Holt and S. Baron-Cohen are joint last authors

${ }^{1}$ Autism Research Centre, Department of Psychiatry, University of Cambridge, Cambridge, UK

Full list of author information is available at the end of the article
}

sensory hypersensitivity [4]. Co-occurring clinical conditions are common and include learning difficulties, sleep disorders and nutritional intolerances [19]. Diagnosis of autism is possible as early as 18 months of age [27]. Autistic traits exist along a spectrum in the wider population [11]. This can be measured in infancy, as demonstrated by the Quantitative Checklist for Autism in Toddlers (Q-CHAT), a novel, dimensional measure of original author(s) and the source, provide a link to the Creative Commons licence, and indicate if changes were made. The images or other third party material in this article are included in the article's Creative Commons licence, unless indicated otherwise in a credit line to the material. If material is not included in the article's Creative Commons licence and your intended use is not permitted by statutory regulation or exceeds the permitted use, you will need to obtain permission directly from the copyright holder. To view a copy of this licence, visit http://creativecommons.org/licenses/by/4.0/. The Creative Commons Public Domain Dedication waiver (http://creativeco mmons.org/publicdomain/zero/1.0/) applies to the data made available in this article, unless otherwise stated in a credit line to the data. 
autistic traits shown to predict later autism diagnosis in several validation studies $[2,3,43]$.

Autism is diagnosed more often in males than in females, despite increasing awareness of its differential presentation in females [33]. The reasons for this gender ratio likely reflect both prenatal biology (genetics and sex steroids) and limitations in current diagnostic methods [32]. In the general population, there are on average, sex differences in specific psychological traits (e.g. systemising and empathising), which are shifted towards a "male" profile in autistic individuals of both genders [8,23].

Several lines of evidence indicate that prenatal sex steroid hormones may be mediating autism likelihood. First, a study of neuroanatomical differences between autistic and neurotypical brains revealed autistic females have atypical structure in regions that substantially overlap with sexually dimorphic regions in neurotypical controls. This suggests that autism affects female brains in regions related to sexual differentiation, which is in turn regulated by prenatal sex steroid hormones [31]. This maleshift is also evident in childhood, as indicated by studies showing that facial features of autistic boys, girls and their siblings are masculinised compared to neurotypical controls $[48,49]$. Autistic males also have higher levels of steroid hormones in amniotic fluid, when these were assessed in a multivariate analysis, as well as higher levels of estrogens in particular in univariate analyses of the same amniotic fluid samples [9, 10]. Additionally, estradiol levels are elevated in maternal serum of pregnancies linked to a later autism diagnosis in the child [13].

Traits and developmental profiles related to autism have also been linked to prenatal sex steroids. For example, prenatal levels of testosterone measured during amniocentesis are negatively correlated with the frequency of eye-contact at 12 months [34] and, vocabulary size at 18 and 24 months [35], and are positively correlated with restricted interests, attention to detail [29] and with autistic traits at 18 and 48 months $[5,6]$.

Epidemiological studies have revealed that maternal polycystic ovary syndrome (PCOS) increases the likelihood of autism in their children $[16,17,30]$. This effect is not primarily driven by shared genetics and is further modulated by the sex of the offspring [15].

PCOS is a complex syndrome affecting between 4 and $20 \%$ of women of reproductive age (depending on the diagnostic criteria), impacting their fertility, metabolism and endocrine regulation [44]. It is particularly associated with higher baseline levels of androgens, as well as with a wider endocrine dysregulation during pregnancy (e.g. on placental function) [37]. Steroidrelated conditions such as PCOS and placental complications are also more common in autistic people $[17,36,40]$, and autistic adults demonstrate signs of steroid imbalance in various tissues [18, 28, 42]. Further research is needed to understand the nature and timing of this endocrine imbalance in autism, as well as to study whether it extends to the wider spectrum of autistic traits in the general population.

The prenatal environment shows baseline sex differences in steroid production via the placenta [21], as well as in markers of placental formation and function, such as human choriogonadotropin (hCG) [1, 20, 38]. Atypical levels in both have been found in maternal serum of pregnancies that resulted in an autistic child, with both higher (for estradiol) and lower levels (for estriol) reported compared to controls $[13,53]$. However, these prenatal factors have not been studied together with fetal sex and in relation to both maternal and infant autistic traits.

To evaluate all these prenatal factors, we assessed both the mothers and their infants for autistic traits [via the Autism Spectrum Quotient (AQ) and Q-CHAT, respectively] in a longitudinal cohort. We then tested whether steroid hormone levels (estradiol, testosterone, DHEAS, progesterone) and placental markers (e.g. hCG) in maternal serum collected during the mother's first ultrasound appointment predicted the autistic traits measured in mothers during pregnancy and in infants at the 18-month follow-up.

\section{Methods \\ Cohort recruitment}

Mothers were recruited during their pregnancy, during or immediately before their routine 20 -week ultrasound scan [mean gestational age of $20.3(\mathrm{SD}=0.4)$ weeks], between 2016 and 2018 at the Rosie Hospital, Cambridge University Hospitals NHS Foundation Trust [7]. This study had been approved by the East of England Cambridge Central Research Ethics Committee (REC Ref 16/EE/0004) and the Research and Development Department of Cambridge University Hospitals. Eligibility inclusion criteria for the study were as follows: (1) little/no consumption of alcohol during pregnancy, (2) no smoking or recreational drug use during pregnancy, and (3) a singleton pregnancy of a fetus (4) whose measurements indicated their size to be appropriate for gestational age [no intrauterine growth restriction (IUGR) or large-for-gestational age (LGA)]. For the postnatal part of the study, the parents of all live singleton births were asked to take part in a series of developmental follow-ups during the first 2 years of life of their infant. Participating mothers also gave informed consent for access to all their pregnancy-related clinical records, test results, and the biological samples that were obtained during their routine clinical care of their pregnancy (both those acquired before as well as after the point of recruitment). 


\section{Clinical data collection}

Serum samples had been collected at the end of the first trimester [mean gestational age of $12.7(\mathrm{SD}=0.7)$ weeks since conception] by a specialist phlebotomist at the Rosie Hospital, Cambridge. These were initially assayed for the levels of human choriogonadotropin (hCG) and pregnancy-associated peptide alpha (PAPP-A) as part of a national screening programme for biomarkers of Down's Syndrome and other conditions, and any remaining serum was stored at $-80{ }^{\circ} \mathrm{C}$. These samples were retrospectively linked to the participating mothers, following their recruitment at approximately 20 -weeks gestation. A total of $n=122$ of these samples were subsequently thawed and transferred to separate vials $(1 \mathrm{ml}$ aliquots per sample), which were further anonymised and sent for additional analysis at the Core Biochemical Assays Laboratory (CBAL) at Addenbrookes Hospital, Cambridge. These corresponded to a subset of the study cohort $(n=219)$ (Fig. 1), since, in many instances, serum had been depleted or discarded after routine prenatal testing prior to recruitment.

In addition, mothers were asked to fill in a Pregnancy History Questionnaire (PHQ) at the point of recruitment [mean gestational age of $20.3(\mathrm{SD}=0.4)$ weeks]. The PHQ is a self-report inventory designed to collect information on metabolic, reproductive and clinically

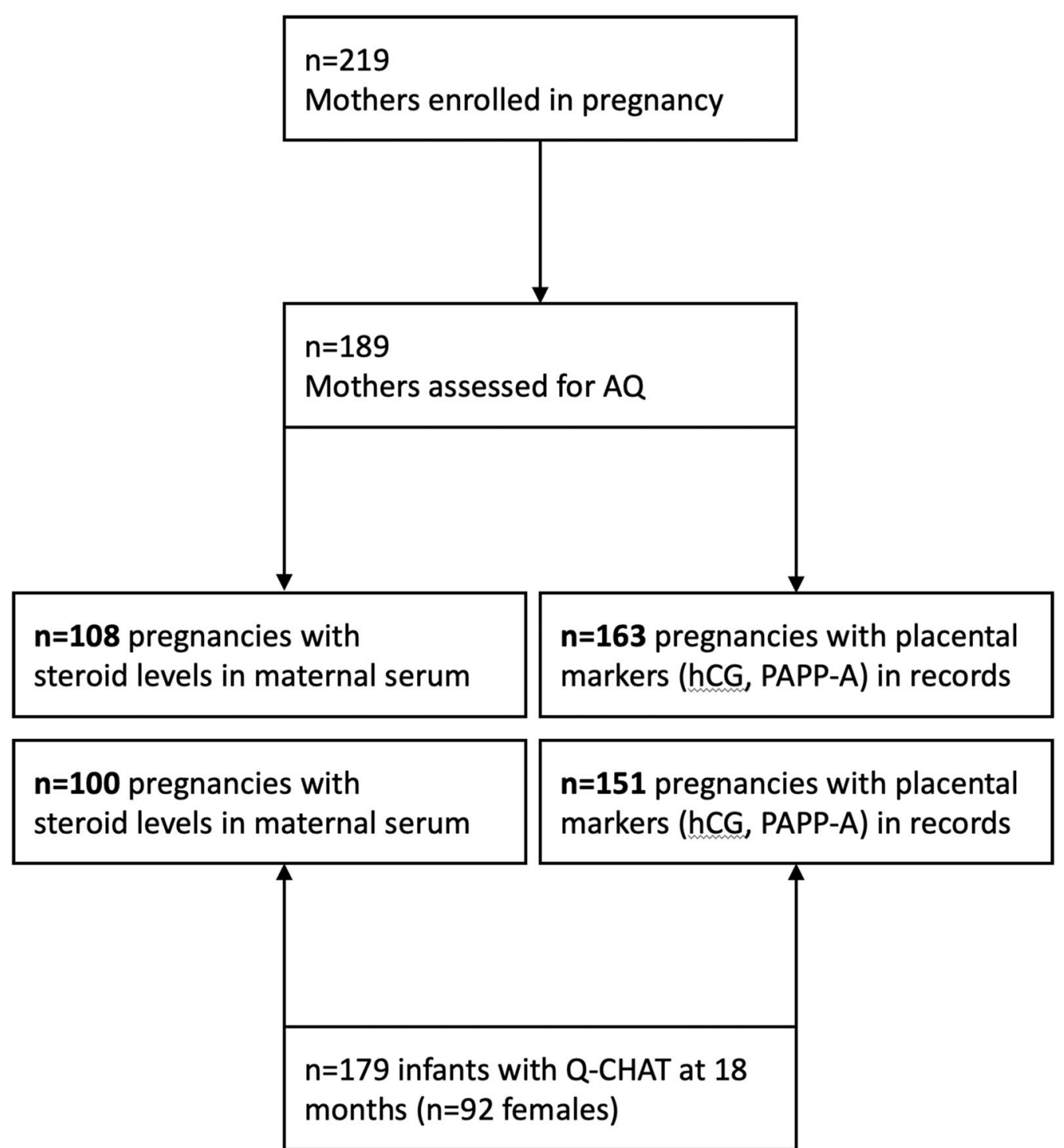

Fig. 1 Flow chart of the study, showing different cohort sizes for each comparison of autistic traits (AQ or Q-CHAT) to prenatal measurements (steroid hormones or placental markers) 
diagnosed conditions of the mothers pertaining to their current pregnancy, as well as those previous. Maternal hirsutism was ascertained by the question 'During your adult years, have you found coarse, dark hair, growing in any of the following areas?', followed by drawings of multiple body areas that are prone to secondary hair growth (e.g. chest, lower face, upper or lower limbs), as described in previous studies [12].

Following labour, birth records containing information on neonate weight and gestational age at birth were also collected.

\section{Additional assays}

The following steroids and peptides were assessed in terms of concentration: Testosterone (T), Estradiol (E2), Dehydroepiandrosterone sulphate (DHEAS), Progesterone (P), sex hormone-binding globulin (SHBG). Samples were analysed on a DiaSorin Liaison ${ }^{\circledR}$ XL automated immunoassay analyser using a one-step competitive chemiluminescence immunoassay for each hormone and two monoclonal antibodies for each peptide. All reagents, standards and consumables are those supplied by DiaSorin [DiaSorin S.p.A, 13040 Saluggia (VC), Italy]. Batch quality precision data and concentration thresholds of detection for each assay are reported in the Additional file 1 (Additional file 1: Table S1).

\section{Autistic traits}

Mothers were asked to complete the Autism Spectrum Quotient (AQ) - Adult version [11] during or immediately after their 20 -week routine ultrasound scan at the Rosie Hospital [mean gestational age of $20.3(\mathrm{SD}=0.4)$ weeks]. In addition, parents were invited via email to complete an online version of the Quantitative Checklist for Autism in Toddlers (Q-CHAT) [3] after their infant reached 18 months of age (mean $=570.6$ days, $\mathrm{SD}=21.8$ days).

All study data were collected and managed using REDCap electronic data capture tools hosted at the University of Cambridge [24, 25].

\section{Statistical analysis}

Autistic trait distributions (maternal AQ and infant Q-CHAT) were assessed for extreme outliers, as defined by an interval of three times the interquartile range. If present, these were reduced to the highest possible value within the interval to facilitate statistical testing.

All steroid hormone concentration values exhibited varying degrees of positive skew and were logtransformed to reduce this and facilitate subsequent statistical testing via linear regression. Following this, outliers were preserved in the analysis to retain clinical heterogeneity. Human choriogonadotropin (hCG) and pregnancy-associated peptide alpha (PAPP-A) values were retrieved from participants' clinical records. These had been standardised according to multiple of the median (MoM) by the Prenatal Screening Department of the Trust according to maternal age, gestational age, and the national means $[46,54]$.

Multivariate analysis was conducted by calculating composite scores for the free fractions of estradiol (E2) and testosterone $(\mathrm{T})$, and by estimating overall steroidogenesis. The free testosterone index (FTI) and free estradiol index (FEI) were calculated via the following formulas (all concentrations in $\mathrm{nmol} / \mathrm{L}$ ) and then logtransformed for further statistical analysis, as previously suggested [45]:

$$
\begin{aligned}
& F E I:(100 *[E 2]) /([S H B G]) \\
& F T I:(100 *[T]) /([S H B G])
\end{aligned}
$$

Latent factor analysis ('nFactors' package) was used to identify the optimal number of common steroidogenic factors based on their correlation matrix. Values for the predicted steroidogenic factor were calculated for each individual via the "Bartlett" method, based on the predicted loadings.

A series of clinical characteristics and group covariates were assessed for pairwise association with autistic traits. In cases of binary traits, differences were tested via Student's $t$-tests. These included comorbidity with PCOS or family history of autism, the latter being defined as present if the participating mothers reported having a first-degree relative (including previous child) that had been diagnosed with autism. A score of clinical severity of maternal hirsutism was devised based on responses in the Pregnancy History Questionnaire (PHQ), which the participating mothers completed following labour. A score of 1 denoted selection of one area of excess hair growth and 2 denoted more than one area. These were further treated as group variables (i.e. "no hirsutism", "one area", "more than one area") and were used in cohort comparisons in terms of hormone levels and autistic traits.

Circulating hormones were log-transformed and assessed for association with autistic traits. For AQ, pairwise Pearson's correlations were first used and then followed-up with a linear regression model with AQ as the outcome variable and the following predictor variables: hormonal concentration, maternal age, comorbidity with PCOS. To account for potential underlying associations between infant sex and Q-CHAT scores, only multiple regression models were used in which an interaction term between infant sex and hormonal concentration was added (for each hormone separately), with Q-CHAT scores as the outcome, and addition of the following covariates: maternal age, maternal PCOS, maternal AQ 
scores, and infant age at assessment adjusted for gestational age at birth. Family history of autism was not included as a covariate in these models, given the very small number of mothers with a diagnosed first-degree relative who also had available serum for hormone assays ( $n=1$ overlap).

The same approach and models were used when examining the hormone composite measures, namely FEI, FTI and the factors defined by unsupervised factor analysis of the steroid hormones.

To ensure their validity, multiple regression models with significant findings for AQ or Q-CHAT were further tested for heteroscedasticity via the studentized Breusch-Pagan test and for the non-normality of their residuals via the Shapiro-Wilk test (Additional file 1: Table S8).

\section{Results}

\section{Cohort characteristics and autistic traits}

Of the $n=219$ pregnant women who consented to take part in the study, $n=17$ had a first-degree relative with autism, $n=26$ had been diagnosed with PCOS, and $n=89$ responded positively to having excess body hair growth in the past. Overall mean age of the mothers was 32.4 years $(\mathrm{SD}=4.54)$. Of this cohort, $n=189$ completed the Autism Spectrum Quotient (AQ), with scores ranging from 1 to 47 (mean =14.63, SD = 8.11) (Fig. 1).

Women with a family history of autism had a significantly higher AQ score $[n=13$, mean $=28(\mathrm{SD}=14.36)]$ compared to women without any first-degree relatives with autism $[n=176$, mean $=13.64(\mathrm{SD}=6.51)]$ (Cohen's $D=1.98, p=0.004$ ) (Table 1). Of the women who replied to both questionnaires, those who reported excess body hair in more than one area of their body $(n=51)$ also had significantly higher AQ scores [mean $=16.8(\mathrm{SD}=6.7)$ ], than those without any sign of excess body hair growth $[n=109, \quad$ mean $=13.8 \quad(\mathrm{SD}=8.9)] \quad($ Cohen's $D=0.36$, $p=0.021$ ) (Fig. 2A). This effect persisted after controlling for family history of autism, maternal age and a diagnosis of PCOS via a linear regression model $[$ Beta $=1.9$ $(\mathrm{SE}=0.67)$, semipartial $r=0.18, p=0.005$ ] (Additional file 1: Table S2).

Of these pregnancies, $n=178$ of the infants were followed-up with the Q-CHAT when they were older than 18 months of age (range 541-671 days after birth), with most being assessed between 18 and 20 months (mean $=570$ days, $\mathrm{SD}=21.3$ days) (Fig. 1 ). Prior to statistical analysis, infant age was adjusted according to the gestational age at birth for each infant (mean $=39.56$, $\mathrm{SD}=1.5$ weeks post-conception).

Regarding Q-CHAT scores, one extreme outlier was noted in the distribution (Q-CHAT $=71$ ), which was reduced to the highest value within an interval of three times the interquartile range $(\mathrm{Q}-\mathrm{CHAT}=53)$, in order to reduce skewness and facilitate statistical comparisons.

There was no significant difference between Q-CHAT scores of male $[$ mean $=30.35(\mathrm{SD}=8.13)]$ and female $[$ mean $=29.63(\mathrm{SD}=7.58)]$ infants at this time-point of assessment (Cohen's $D=0.09, p=0.54$ ).

Q-CHAT scores were significantly correlated with maternal AQ scores (Pearson's $r=0.21, p=0.008$ ) (Additional file 1: Fig. S1).

\section{Hormone covariates and factor analysis}

Hormone concentrations were only available in subsets of the cohort of women that consented to the study. This differed slightly for placental markers that were part of routine prenatal screening (hCG and PAPP-A) and steroid level measurements, which were analysed for research purposes on the remaining serum sample for each participant (Fig. 1).

The analysed maternal serum samples corresponded to a narrow period of gestation between the late first and early second trimester (mean $=12.7$ weeks, $\mathrm{SD}=0.8$ weeks). Circulating hormones showed varying degrees of correlation with each other (Fig. 3) and with other demographic and clinical variables. Testosterone, DHEAS and progesterone were all positively correlated with maternal age (Additional file 1: Table S3). Unsupervised factor analysis showed that a common latent factor could be derived from estradiol, testosterone and DHEAS, which account for $32 \%$ of the total variance in hormone levels. The values for this 'steroidogenic factor' were estimated for each participant based on the factor loadings and steroid concentrations (Additional file 1: Figure S2).

Women with PCOS also had significantly higher levels of estradiol and progesterone, but lower levels of SHBG, compared to women without the condition (Additional file 1: Table S4). Maternal age correlated positively with progesterone, but negatively with testosterone, FTI and DHEAS (Additional file 1: Table S4). Women with a history of hirsutism in more than one area of their body also had significantly higher levels of estradiol (Cohen's $D=0.47, p=0.034$ ) and FEI (Cohen's $D=0.51, p=0.025$ ), as well as higher predicted values for the latent steroid factor $(D=0.50, p=0.028)$ (Fig. 2B).

\section{Associations between hormones and maternal AQ score}

The association between hormones in maternal serum and maternal autistic traits was investigated via univariate Pearson's correlation coefficient and with multiple regression (MR) controlled for maternal age and a diagnosis of PCOS (Table 2). FEI was significantly correlated with maternal AQ score using both methods (MR: semipartial $r=0.19, p=0.048$ ) (Fig. 4A). Estradiol levels were 


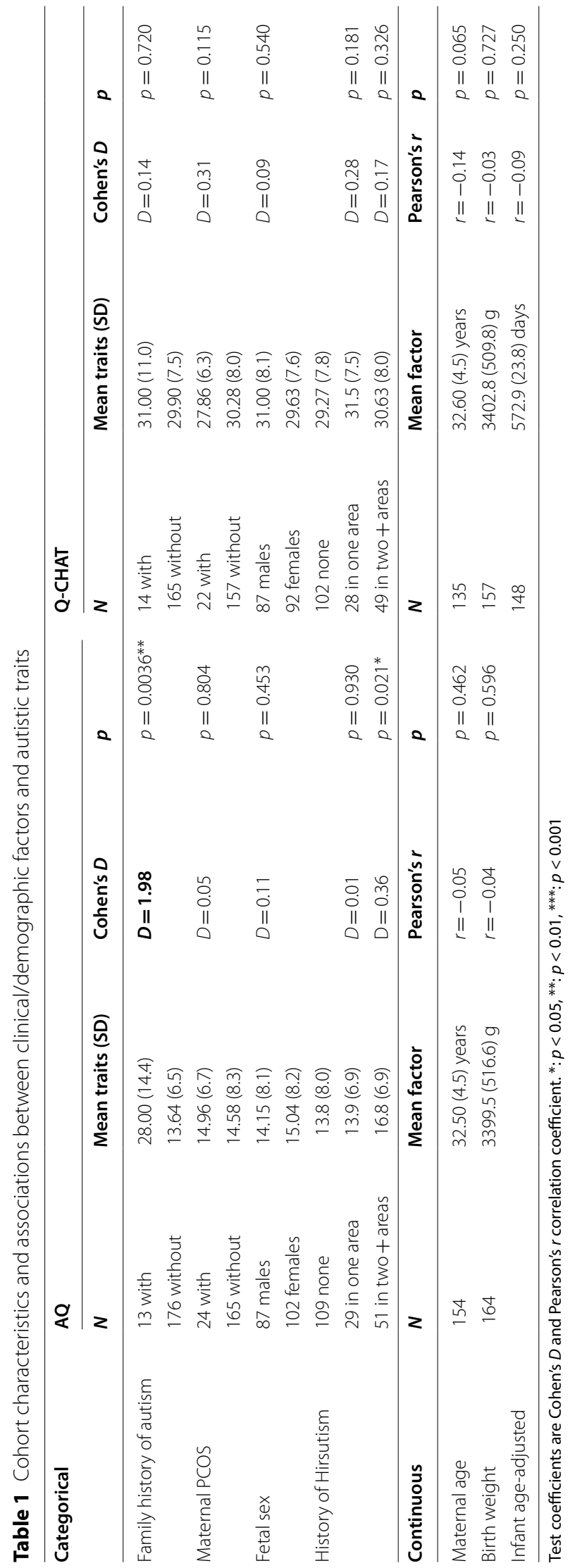



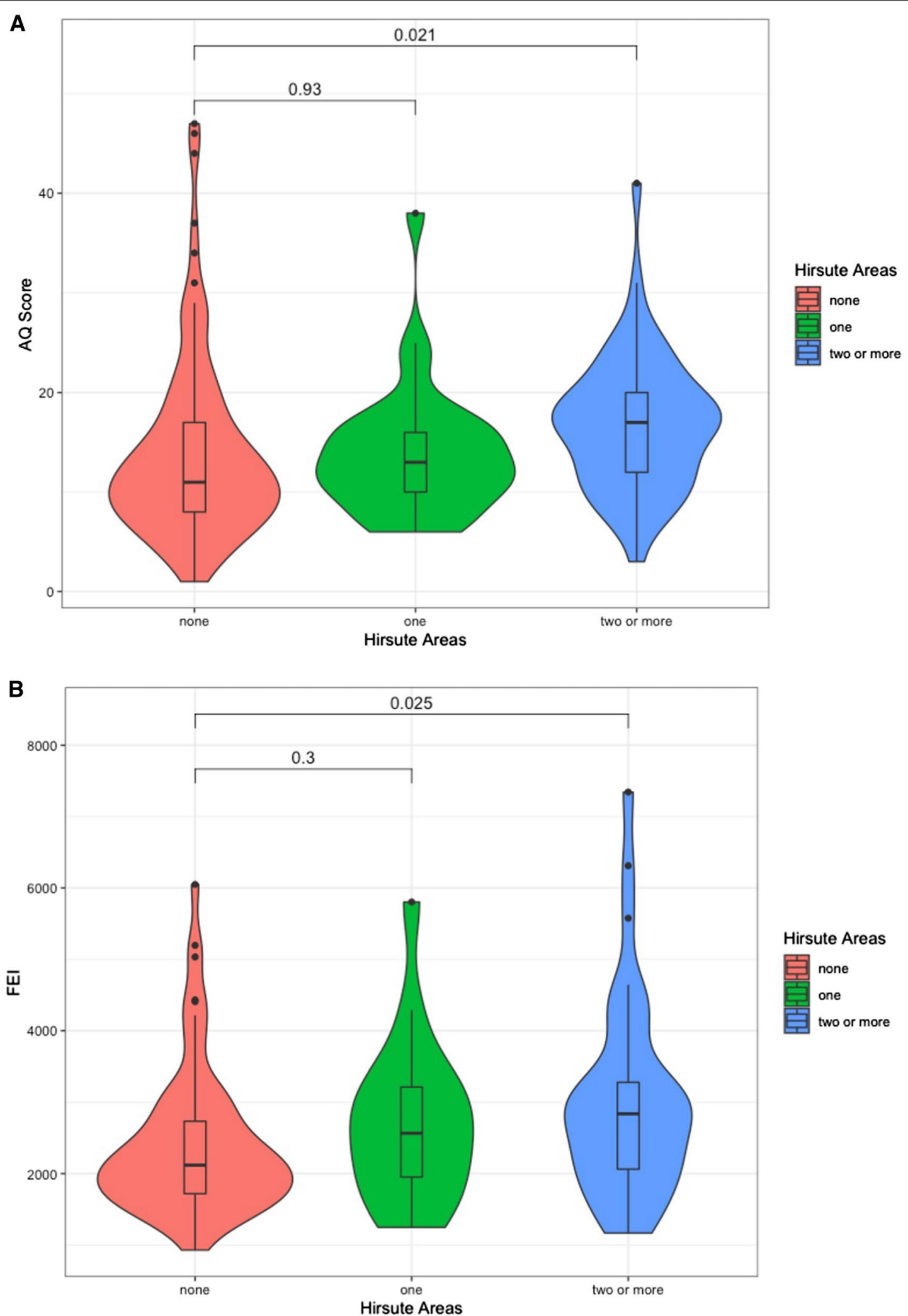

Fig. 2 Violin-boxplots showing the distribution of $\mathbf{A}$ maternal autistic traits $(A Q)$ and $\mathbf{B}$ free estradiol levels (FEl), according to clinical history of hirsutism. Women that reported two or more body areas affected had significantly higher levels of both AQ $(p=0.021)$ and FEl $(p=0.025)$ 
associated with AQ in Pearson's correlation $(r=0.20$, $p=0.036$ ), but this was not statistically significant in the multiple regression model that controlled for other covariates (MR: semipartial $r=0.11, p=0.25$ ).

\section{Associations between hormones and infant Q-CHAT scores} Log-transformed concentrations of hormone levels were studied in relation to infant Q-CHAT with multiple linear regression models that controlled for birth weight, maternal $\mathrm{PCOS}$, maternal $\mathrm{AQ}$ and infant age at the time of Q-CHAT, adjusted for gestational age at birth. The interaction between hormone concentration and infant sex was also used as a variable in the same model (Table 3). Infant sex moderated the association between maternal estradiol levels and Q-CHAT scores, with a positive association for Q-CHAT in males but not females (hormone-by-sex: semipartial $r=0.23, p=0.036$ ) (Table 3; Fig. 4B).

Standardised hCG MoM values were significantly associated with Q-CHAT scores, with a significant moderating effect for infant sex (hormone-by-sex: semipartial $r=-0.26, p=0.005)$. This effect followed the opposite pattern to estradiol, with a positive relationship in females but not males (Table 3).
All multiple regression models that yielded significant results (for both AQ and Q-CHAT) passed tests on the assumptions of homoscedasticity and normality of residuals (full model results: Additional file 1: Tables S6-S8).

\section{Discussion}

This is the first clinical longitudinal study to report on the endocrine profile of pregnant women and how this relates to their own autistic traits and to the autistic traits of their infants. First, we found that the fraction of free circulating estradiol (FEI) during pregnancy correlated positively with the autistic traits of pregnant neurotypical women. Second, we found that maternal estradiol was associated with infant autistic traits (as measured by the Q-CHAT) in a sex-dependent way, with a positive correlation in males but not females. Third, we found that the opposite was true for standardised hCG MoM values, where a negative correlation with Q-CHAT scores in males was noted. These associations with early neurodevelopment were independent of the mother's $A Q$ score or PCOS status, as well as other infant characteristics such as birth weight or age at the time of assessment. Finally, this is the first longitudinal study to show a significant positive correlation between maternal and infant autistic traits, as measured by the AQ and Q-CHAT, respectively.

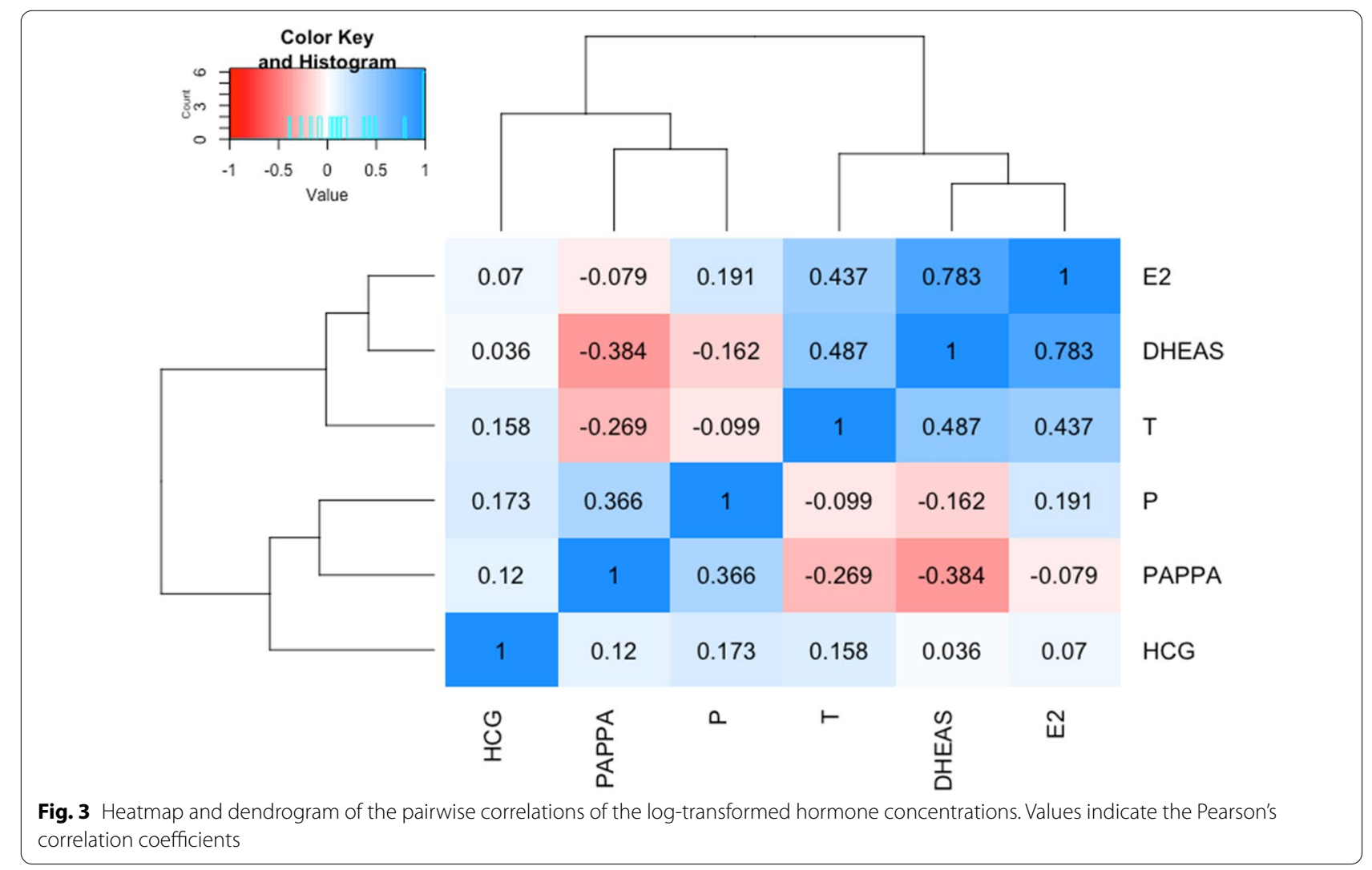


Table 2 Pearson's correlation (hormone and $A Q$ ) and multiple regression models (MR) for predicting $A Q$ scores, controlled for maternal age and a diagnosis of PCOS

\begin{tabular}{|c|c|c|c|c|}
\hline & Test coefficient & SE & $\begin{array}{l}\text { Semipartial } \\
\text { correlation }\end{array}$ & $p$ value \\
\hline \multicolumn{5}{|l|}{ Estradiol } \\
\hline Pearson's & $r=0.20$ & 0.09 & & $p=0.036^{*}$ \\
\hline MR: intercept & Bet $a=4.52$ & 12.29 & & $p=0.714$ \\
\hline Predictor & Bet $a=1.43$ & 1.23 & $r=0.11$ & $p=0.250$ \\
\hline \multicolumn{5}{|l|}{ Testosterone } \\
\hline Pearson's & $r=0.09$ & 0.10 & & $p=0.377$ \\
\hline MR: intercept & Beta $=17.34$ & 4.50 & & $p<0.001^{* * *}$ \\
\hline Predictor & Beta $=0.34$ & 1.12 & $r=0.03$ & $p=0.761$ \\
\hline \multicolumn{5}{|l|}{ DHEAS } \\
\hline Pearson's & $r=0.09$ & 0.10 & & $p=0.379$ \\
\hline MR: intercept & Beta $=15.29$ & 6.47 & & $p=0.020^{*}$ \\
\hline Predictor & Bet $a=0.44$ & 0.86 & $r=0.05$ & $p=0.600$ \\
\hline \multicolumn{5}{|l|}{ Progesterone } \\
\hline Pearson's & $r=-0.09$ & 0.09 & & $p=0.365$ \\
\hline$M^{a}$ : intercept & Beta $=23.23$ & 6.94 & & $p=0.001^{* *}$ \\
\hline Predictor & Bet $a=-2.16$ & 2.22 & $r=-0.09$ & $p=0.334$ \\
\hline \multicolumn{5}{|l|}{ hCGMOM } \\
\hline Pearson's & $r=-0.03$ & 0.08 & & $p=0.665$ \\
\hline MR: intercept & Beta $=13.92$ & 0.97 & & $p<0.001^{* * *}$ \\
\hline Predictor & Bet $a=-0.27$ & 0.58 & $r=-0.04$ & $p=0.650$ \\
\hline \multicolumn{5}{|l|}{ PAPP-AMOM } \\
\hline Pearson's & $r=-0.04$ & 0.08 & & $p=0.589$ \\
\hline MR : intercept & Beta $=14.18$ & 1.16 & & $p<0.001^{* * *}$ \\
\hline Predictor & Bet $a=-0.49$ & 0.84 & $r=-0.05$ & $p=0.560$ \\
\hline \multicolumn{5}{|c|}{$\begin{array}{l}\text { Composite meas- } \\
\text { ures }\end{array}$} \\
\hline \multicolumn{5}{|c|}{ Free Estradiol Index } \\
\hline Pearson's & $r=0.22$ & 0.09 & & $p=0.019^{*}$ \\
\hline MR: intercept & Beta $=13.76$ & 4.61 & & $p=0.004^{* *}$ \\
\hline Predictor & Bet $a=2.76$ & 1.4 & $r=0.19$ & $p=0.048^{*}$ \\
\hline \multicolumn{5}{|l|}{$\begin{array}{l}\text { Free Testosterone } \\
\text { Index }\end{array}$} \\
\hline Pearson's & $r=0.11$ & 0.10 & & $p=0.258$ \\
\hline MR: intercept & Beta $=17.48$ & 4.21 & & $p<0.001$ \\
\hline Predictor & Bet $a=0.69$ & 4.21 & $r=0.07$ & $p=0.484$ \\
\hline \multicolumn{5}{|l|}{ Steroid factor } \\
\hline Pearson's & $r=0.18$ & 0.10 & & $p=0.061$ \\
\hline MR: intercept & Beta $=16.75$ & 0.09 & & $p<0.001^{* * *}$ \\
\hline Predictor & Bet $a=0.77$ & 0.55 & $r=0.17$ & $p=0.085$ \\
\hline
\end{tabular}

The association between free circulating estradiol and maternal AQ was independent of maternal age or a diagnosis of PCOS. In addition, women with clinical history of excess body hair growth in more than one area of their body also had significantly higher autistic traits scores, as well as higher levels of circulating estradiol and
FEI (Fig. 2). This is consistent with previous studies that reported higher rates of steroid-exposure related symptoms, such as hirsutism in autistic women [40]. The correlation between FEI and AQ was independent of PCOS diagnostic status, and women diagnosed with PCOS did not have higher AQ scores (Table 1). This may be due to reduced statistical power $(n=13$ with PCOS diagnosis) or the fact that all the women in this cohort had no autism diagnosis themselves and were without significant challenges in fertility, contrary to previous studies that found a link between PCOS and autism [17].

There was no association between testosterone levels or the FTI and maternal autistic traits in this study. Testosterone correlated positively with estradiol (Additional file 1: Table S3) but did not differ between women with and without PCOS, or correlate with a clinical history of hirsutism. Previous studies have also shown that circulating testosterone levels measured at a single time-point do not always correlate closely with their associated clinical parameters, such as hair growth [39]. Particularly during pregnancy, estradiol levels may be more clinically informative, as testosterone is rapidly aromatised into estradiol by the placenta. Estradiol could then be interpreted as the end-product of wider steroidogenesis and may be a better biomarker of the 'steroidopathy' previously indicated by epidemiological studies of autistic women [40].

This is also the first study to investigate circulating hormones in maternal serum in relation to autistic traits in their infants measured between 18 and 20 months of age via the Q-CHAT. This questionnaire was developed to enable parents to quantify autistic traits, moving away from a restrictive binary format of items toward a Likert format. To date, the Q-CHAT has been validated in several studies. Interestingly, the Q-CHAT also shows sex differences in early autistic traits, with males scoring significantly higher than females in larger cohorts $[2,3,41$, 43].

The current study found significant moderation effects of infant sex on the associations between hormones and neurodevelopment. Specifically, increased maternal estradiol was more predictive of Q-CHAT scores of males than females (Fig. 4; Table 3). This was independent of potential confounding variables, such as maternal age, diagnosis of PCOS and maternal autistic traits. Contrary to the results with the $\mathrm{AQ}$, free estradiol levels (FEI), as estimated via serum SHBG, were not predictive of Q-CHAT scores. SHBG is a peptide, and it does not cross the placenta as easily as steroids, as shown in rare cases of partial deficiency in mothers but not their fetus [26]. Maternal SHBG may therefore not accurately capture the bioavailability of steroids in the fetal circulation nor accurately predict potential effects on infant neurodevelopment. 
A.

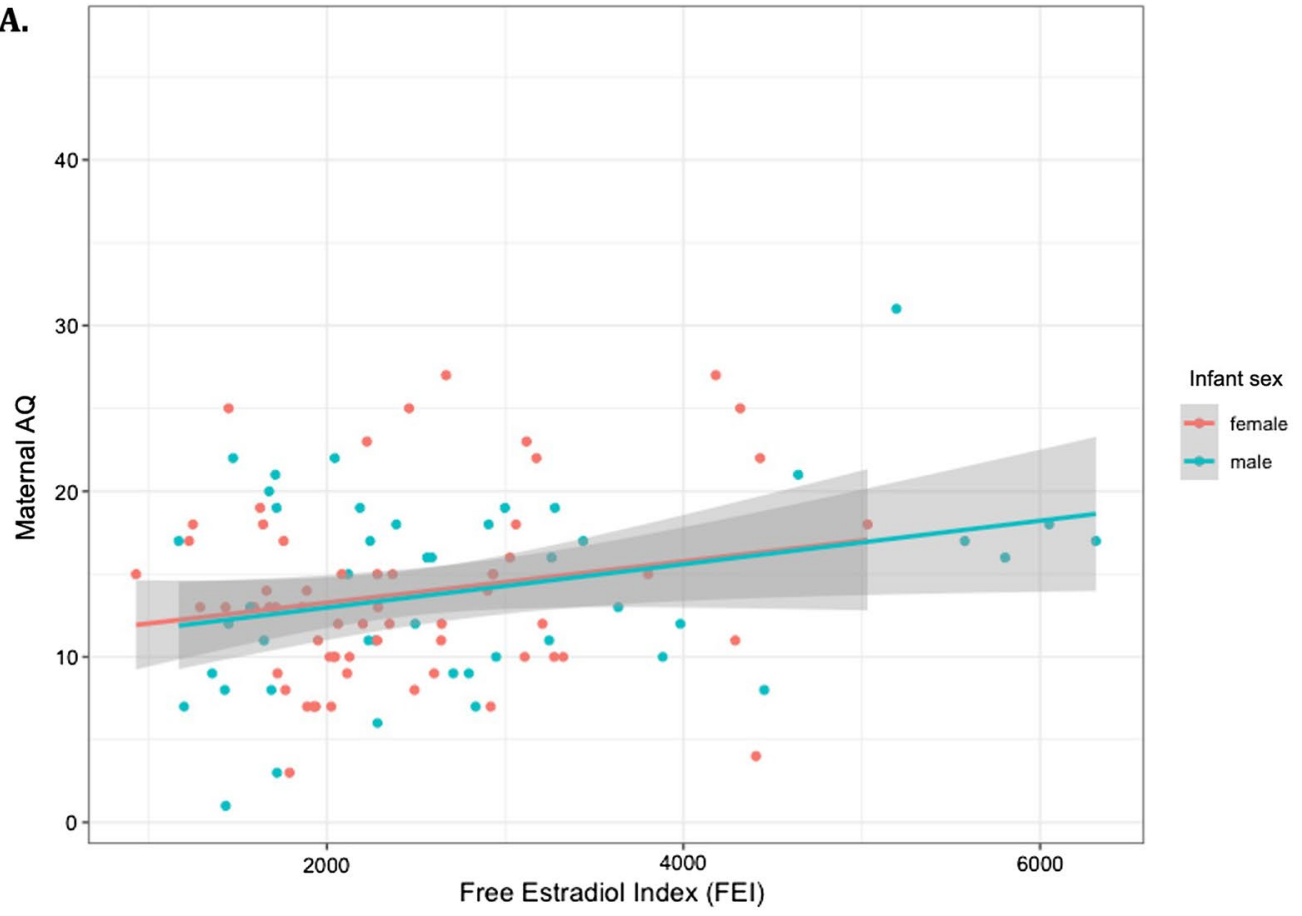

B.

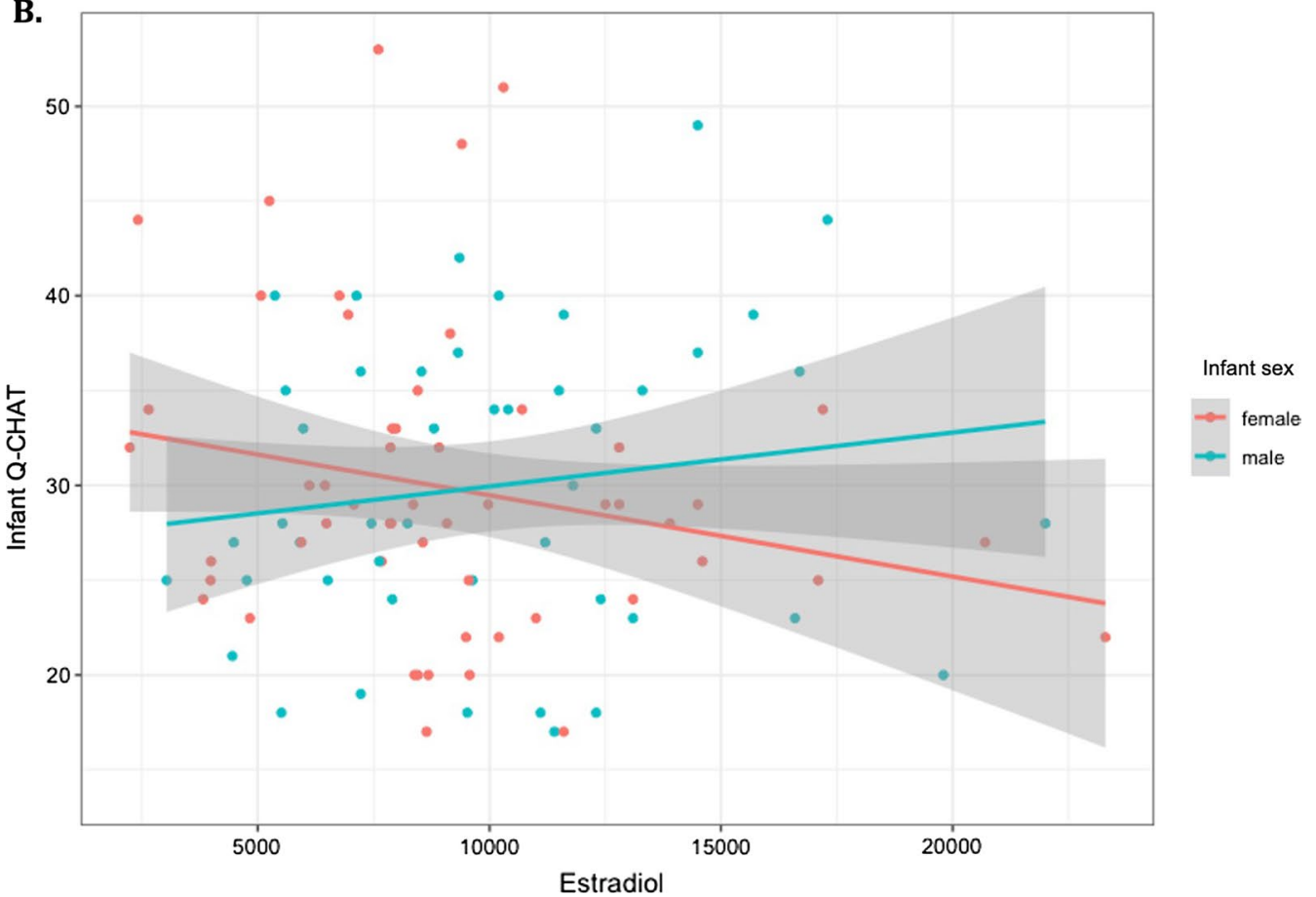

Fig. 4 Scatterplots with linear fit-models for $\mathbf{A}$ the association between maternal free estradiol (FEI) and AQ score, and $\mathbf{B}$ the association between maternal estradiol and Q-CHAT; separate linear models are presented for each sex and show significant interactions with infant sex in the case of infant Q-CHAT

Further research is needed to understand the interaction between maternal estradiol levels and fetal sex in predicting Q-CHAT scores. As with the observed male bias in autism diagnoses, the lack of an association 
Table 3 Multiple regression models for predicting Q-CHAT scores and accounting for a hormone-by-sex interaction, further controlled for maternal $\mathrm{AQ}$, a diagnosis of $\mathrm{PCOS}$, maternal age, birth weight and infant age at Q-CHAT assessment-adjusted for gestational age at birth

\begin{tabular}{lll}
\hline Q-CHAT & \\
\cline { 2 - 3 } $\begin{array}{l}\text { Regression } \\
\text { coefficient }\end{array}$ & SE & $\begin{array}{l}\text { Semipartial } p \text { value } \\
\text { correlation } \\
\text { coefficient }\end{array}$ \\
\hline
\end{tabular}

\begin{tabular}{|c|c|c|c|c|}
\hline \multicolumn{5}{|l|}{ Estradiol } \\
\hline MR: intercept & Beta $=114.20$ & 35.21 & & $p=0.002^{* *}$ \\
\hline Hormone & Beta $=-4.00$ & 2.311 & -0.19 & $p=0.087$ \\
\hline Hormone-by-sex & Beta $=8.27$ & 4.08 & 0.23 & $p=0.036^{*}$ \\
\hline \multicolumn{5}{|l|}{ Testosterone } \\
\hline MR: intercept & Beta $=80.01$ & 29.07 & & $p=0.007^{* *}$ \\
\hline Hormone & Beta $=-1.95$ & 2.12 & -0.10 & $p=0.362$ \\
\hline Hormone-by-sex & Beta $=0.99$ & 3.28 & 0.03 & $p=0.765$ \\
\hline \multicolumn{5}{|l|}{ DHEAS } \\
\hline MR: intercept & Beta $=90.03$ & 29.68 & & $p=0.003^{* *}$ \\
\hline Hormone & Bet $a=-2.33$ & 1.58 & -0.16 & $p=0.144$ \\
\hline Hormone-by-sex & Bet $a=1.36$ & 3.22 & 0.04 & $p=0.674$ \\
\hline \multicolumn{5}{|l|}{ Progesterone } \\
\hline MR : intercept & Beta $=84.06$ & 32.16 & & $p=0.011^{*}$ \\
\hline Hormone & Beta $=-3.70$ & 4.65 & -0.08 & $p=0.429$ \\
\hline Hormone-by-sex & Beta $=11.64$ & 6.86 & 0.18 & $p=0.095$ \\
\hline \multicolumn{5}{|l|}{ hCGMoM } \\
\hline MR: intercept & Beta $=34.2$ & 20.61 & & $p=0.100$ \\
\hline Hormone & Beta $=2.95$ & 1.07 & 0.24 & $p=0.007^{* *}$ \\
\hline Hormone-by-sex & Beta $=-4.34$ & 1.53 & -0.25 & $p=0.005^{* *}$ \\
\hline \multicolumn{5}{|l|}{ PAPP-A MOM } \\
\hline MR: intercept & Beta $=46.04$ & 21.13 & & $p=0.031^{*}$ \\
\hline Hormone & Beta $=1.69$ & 1.45 & 0.11 & $p=0.245$ \\
\hline Hormone-by-sex & Bet $a=-1.37$ & 2.29 & -0.05 & $p=0.552$ \\
\hline \multicolumn{5}{|l|}{$\begin{array}{l}\text { Composite meas- } \\
\text { ures }\end{array}$} \\
\hline \multicolumn{5}{|l|}{ Free Estradiol Index } \\
\hline MR: intercept & Beta $=82.42$ & 29.02 & & $p=0.006^{* *}$ \\
\hline Hormone & Beta $=-0.969$ & 1.37 & -0.07 & $p=0.481$ \\
\hline Hormone-by-sex & Beta $=1.989$ & 1.68 & 0.13 & $p=0.240$ \\
\hline \multicolumn{5}{|l|}{$\begin{array}{l}\text { Free Testosterone } \\
\text { Index }\end{array}$} \\
\hline MR: intercept & Beta $=77.86$ & 29.16 & & $p=0.009^{* *}$ \\
\hline Hormone & Beta $=2.50$ & 8.78 & $r=0.03$ & $p=0.777$ \\
\hline Hormone-by-sex & Bet $a=-9.53$ & 11.69 & $r=-0.09$ & $p=0.417$ \\
\hline \multicolumn{5}{|l|}{ Steroid factor } \\
\hline MR: intercept & Beta $=85.95$ & 29.01 & & $p=0.004^{* *}$ \\
\hline Hormone & Bet $a=-1.95$ & 1.20 & $r=-0.17$ & $p=0.108$ \\
\hline Hormone-by-sex & Beta $=2.80$ & 1.81 & $r=0.16$ & $p=0.126$ \\
\hline
\end{tabular}

${ }^{*}: p<0.05,{ }^{* *}: p<0.01{ }^{* * *}: p<0.001$ between hormone levels in females may reflect behavioural differences that are not adequately captured by this particular instrument [32]. Alternatively, this interaction may represent evidence of differential liability to prenatal hormone exposure, whereby males are affected more than females by the same maternal endocrine factors. Since males are undergoing an additional increase in steroid levels during mid-pregnancy, due to the activation of the testes, this interaction could also be attributed to the added effect of elevated steroids in the fetal circulation of males [51]. While fetal steroids were not measured directly in this study, previous comparisons in humans showed that estradiol levels correlated between maternal serum and amniotic fluid, in both the second and third trimesters [50]. The same was found for hCG levels in the second trimester [47]. In contrast, increases in androgen levels of male pregnancies were not detectable in maternal serum [50]. Therefore, the interaction of sex with estradiol levels in predicting Q-CHAT scores, in this study, could potentially be attributed to the additive effect of elevated androgens in the fetal circulation of males, which may not have been captured by assaying maternal serum, rather than amniotic fluid [5].

The findings with hCG mirror those for estradiol, showing a negative association with Q-CHAT scores in females but not males. hCG is produced by the developing trophoblast cells in the placenta and regulates early implantation as well as steroid production [14]. hCG levels also show baseline sex differences in typical cohorts as early as the first half of pregnancy [1]. In the current cohort, hCG was measured late in the first trimester, during the first ultrasound visit, as part of the screening programme for Down's syndrome. This is part of routine prenatal screening in the UK to test for placental dysfunction that can often be indicative of genomic instability due to aneuploidies [54]. In cases of clinically diagnosed autism, both very low and very high levels of hCG have been found in maternal serum, leading to a "U-shaped" association when studying both males and females [53]. Furthermore, autistic traits in the children have been associated with the severity of nausea and 'morning sickness' during pregnancy, symptoms that have also been linked to high hCG levels [22, 52]. Further studies into placental functionality could offer insight into these observations, the role of sex, and more specifically, whether the observed interaction effect for Q-CHAT score is part of an adaptive response that is more pronounced in females than males. 


\section{Limitations}

Limitations of the current study include the relatively small sample size, as well as potential ascertainment bias given the voluntary process of recruitment. In addition, AQ and Q-CHAT scores were both rated by the mother herself. This was addressed by controlling for maternal AQ when studying associations between hormones and infant Q-CHAT scores. The items of the AQ-Adult and Q-CHAT are also substantially different, with the latter dealing with behavioural and developmental milestones that are specific to infants rather than to interests and personality traits that are more evident in adulthood. Furthermore, the findings of the current study may have been inflated by Type I errors, as $p$ value thresholds were not corrected for multiple testing. However, the findings on estradiol were consistent for both maternal and infant outcomes (Fig. 4), were reflected in clinical hirsutism differences (Fig. 2), and are in accordance with those observed in other studies of clinical autism [10,13]. The high degree of correlation between many of the assessed hormones (Fig. 3) also indicates a common functional and regulatory framework. The association tests for individual hormones may therefore not be entirely independent, but instead affected by a common steroidogenic factor as previously reported [9]. Replication of these findings in a larger, independent cohort is warranted to confirm their validity.

\section{Conclusions}

This is the first longitudinal study to report associations between maternal steroidogenic factors and the autistic traits of both the mother and her infant, with significant moderating effects of sex being noted for the latter. Additional research is needed to replicate these findings, to establish how maternal steroidogenesis may affect fetal neurodevelopment, and to determine how these processes interact with genetics to disproportionately increase the liability for autism in males.

\begin{abstract}
Abbreviations
AQ: Autism Spectrum Quotient; BDNF: Brain-derived neurotrophic factor; CBAL: Core Biochemical Assays Laboratory; CUSP: Cambridge Ultrasound Siblings and Parents Project; DHEAS: Dehydroepiandrosterone sulphate; E2: Estradiol; FEl: Free Estradiol Index; FTI: Free Testosterone Index; hCG: Human chorionic gonadotropin; IUGR: Intrauterine growth restriction; LGA: Large for gestational age; MoM: Multiple of the median; P: Progesterone; PAPP-A: Pregnancy-associated peptide alpha; PCOS: Polycystic ovary syndrome; PHQ: Pregnancy History Questionnaire; Q-CHAT: Quantitative Checklist of Autism in Toddlers; SD: Standard deviation; SHBG: Sex hormone binding globulin.
\end{abstract}

\section{Supplementary Information}

The online version contains supplementary material available at https://doi. org/10.1186/s13229-021-00453-7.

Additional file 1. Supplementary Figures and Tables.

\section{Acknowledgements}

The authors would like to express their gratitude to Sally Goodburn (Prenatal Screening Unit), Keith Burling and Peter Barker (Core Biochemical Assay Laboratory) for their assistance with serum sample handling, delivery, and the hormonal assays. In addition, we wish to thank Dr Simon R White for his statistical advice and support.

\section{Authors' contributions}

AT conducted the analysis, interpreted the data and drafted the manuscript. EA and CA contributed significantly to the organisation and design of the study and to data acquisition. EP and GR helped with the statistical analysis and interpretation of the data. GH and TA provided guidance with and supervised the use of clinical data. SBC and RH contributed equally to study design, study supervision, data interpretation, and to the revisions of the manuscript. All authors read and approved the final manuscript.

\section{Funding}

This particular research was funded in part by the Wellcome Trust (GRANT No. RNAG/528, block code: 214322 Z\\18\Z). Additional funds were provided by the Autism Research Trust (ART, registered charity number: 1136737,

UK) via purpose-specific Grants (RNAG/425) for the conducting of clinical studies relating to early autism biomarkers. For the purpose of Open Access, the authors have applied a CC BY public copyright licence to any Author Accepted Manuscript version arising from this submission. AT is further funded by the Peterhouse Graduate Studentship. SBC is funded by the Autism Research Trust, the Templeton World Charitable Foundation, and the NIHR Biomedical Research Centre in Cambridge, during the period of this work. SBC also receives funding from the Innovative Medicines Initiative 2 Joint Undertaking (JU) under Grant Agreement No. 777394. The JU receives support from the European Union's Horizon 2020 research and innovation programme and EFPIA, AUTISM SPEAKS, Autistica, and SFARI. His research is also supported by the National Institute of Health Research (NIHR) Applied Research Collaboration East of England (ARC EoE) programme. The views expressed are those of the authors, and not necessarily those of the NIHR, NHS or Department of Health and Social Care.

\section{Availability of data and materials}

The datasets generated during and/or analysed during the current study are not publicly available due to limited ethics approval for the wider clinical study (CUSP) by CUH and to the specific consent provided by the participants. They may be available from the corresponding author on reasonable request and pending approval of any future analyses by $\mathrm{CUH}$.

\section{Declarations}

\section{Ethics approval and consent to participate}

A favourable ethical opinion for the study's protocol, recruitment criteria and related materials, including the consent form, was given by the East of England Cambridge Central Research Ethics Committee (REC Ref 16/EE/0004) and the Research and Development Department of Cambridge University Hospitals.

\section{Consent for publication}

Not applicable.

\section{Competing interests}

The authors declare that they have no competing interests.

\section{Author details}

${ }^{1}$ Autism Research Centre, Department of Psychiatry, University of Cambridge, Cambridge, UK. ${ }^{2}$ Department of Clinical Psychological Science, Faculty of Psychology and Neuroscience, Maastricht University, Maastricht, The Netherlands. ${ }^{3}$ School of Psychology, Faculty of Medical Sciences, Newcastle University, Newcastle upon Tyne, UK. ${ }^{4}$ The Rosie Hospital, Cambridge University Hospitals Foundation Trust, Cambridge, UK.

Received: 21 January 2021 Accepted: 15 June 2021

Published online: 08 July 2021 


\section{References}

1. Adibi JJ, Lee MK, Saha S, Boscardin WJ, Apfel A, Currier RJ. Fetal sex differences in human chorionic gonadotropin fluctuate by maternal race, age, weight and by gestational age. J Dev Orig Health Dis. 2015. https://doi. org/10.1017/S2040174415001336

2. Allison C, Mathew FE, Ruta L, Pasco G, Soufer R, Brayne C, Charman T, Baron-Cohen S. The Quantitative Checklist for Autism in Toddlers (Q(HAT). A population screening study with follow-up: the case for multiple time-point screening for autism. BMJ Open Paediatr. 2021:5:e000700.

3. Allison C, Baron-Cohen S, Wheelwright S, Charman T, Richler J, Pasco G, Brayne C. The Q-CHAT (Quantitative CHecklist for Autism in Toddlers): a normally distributed quantitative measure of autistic traits at 18-24 months of age: preliminary report. J Autism Dev Disord. 2008;38(8):141425. https://doi.org/10.1007/s10803-007-0509-7.

4. Association, American Psychiatric. Autism Spectrum Disorder 299.00 (F84.0). In: Diagnostic and statistical manual of mental disorders, 5th ed. 2013; Washington.

5. Auyeung B, Taylor K, Hackett G, Baron-Cohen S. Foetal testosterone and autistic traits in 18 to 24-month-old children. Mol Autism. 2010;1(1):11. https://doi.org/10.1186/2040-2392-1-11.

6. Auyeung B, Baron-Cohen S, Ashwin E, Knickmeyer R, Taylor K, Hackett G. Fetal testosterone and autistic traits. Br J Psychol. 2009. https://doi.org/10. 1348/000712608X311731.

7. Aydin E, Holt R, Chaplin D, Hawkes R, Allison C, Hackett G, Austin T, et al. Fetal anogenital distance using ultrasound. Prenat Diagn. 2019;39(7):527-35. https://doi.org/10.1002/pd.5459.

8. Baron-Cohen S, Cassidy S, Auyeung B, Allison C, Achoukhi M, Robertson S, Pohl A, Lai MC. Attenuation of typical sex differences in 800 adults with autism vs. 3,900 controls. PLoS ONE. 2014;9(7): e102251. https://doi.org/10.1371/journal.pone.0102251.

9. Baron-Cohen S, Bonnie Auyeung B, Nørgaard-Pedersen DM, Hougaard MW, Abdallah LM, Cohen AS, Chakrabarti B, Ruta L, Lombardo MV. Elevated fetal steroidogenic activity in autism. Mol Psychiatry. 2015;20(June):369-76. https://doi.org/10.1038/mp.2014.48.

10. Baron-Cohen S, Tsompanidis A, Auyeung B, Nørgaard-Pedersen B, Hougaard DM, Abdallah M, Cohen A, Pohl A. Foetal oestrogens and autism. Mol Psychiatry. 2019. https://doi.org/10.1038/s41380-019-0454-9.

11. Baron-Cohen S, Wheelwright S, Skinner R, Martin J, Clubley E. The Autism-Spectrum Quotient (AQ): evidence from asperger syndrome/ high-functioning autism, males and females, scientists and mathematicians. J Autism Dev Disord. 2001. https://doi.org/10.1023/A:10056 53411471.

12. Barrett ES, Hoeger KM, Sathyanarayana S, Abbott DH, Redmon JB, Nguyen RHN, Swan SH. Anogenital distance in newborn daughters of women with polycystic ovary syndrome indicates fetal testosterone exposure. J Dev Orig Health Dis. 2018. https://doi.org/10.1017/S2040 174417001118.

13. Bilder DA, Sean Esplin M, Coon H, Burghardt P, Clark EAS, Fraser A, Smith $\mathrm{KR}$, et al. Early second trimester maternal serum steroid-related biomarkers associated with autism spectrum disorder. J Autism Dev Disord. 2019. https://doi.org/10.1007/s10803-019-04162-2.

14. Canfield RE, O'Connor JF, Birken S, Krichevsky A, Wilcox AJ. Development of an assay for a biomarker of pregnancy and early fetal loss. Environ Health Perspect. 1987. https://doi.org/10.1289/ehp.877457.

15. Cesta CE, Öberg AS, Ibrahimson A, Yusuf I, Larsson H, Almqvist C, D'Onofrio BM, et al. Maternal polycystic ovary syndrome and risk of neuropsychiatric disorders in offspring: prenatal androgen exposure or genetic confounding? Psychol Med. 2020. https://doi.org/10.1017/S0033 291719000424

16. Chen X, Kong L, Piltonen TT, Gissler M, Lavebratt C. Association of polycystic ovary syndrome or anovulatory infertility with offspring psychiatric and mild neurodevelopmental disorders: a Finnish population-based cohort study. Hum Reprod. 2020. https://doi.org/10.1093/humrep/deaa1 92

17. Cherskov A, Pohl A, Allison C, Zhang H, Payne RA, Baron-Cohen S. Polycystic ovary syndrome and autism: a test of the prenatal sex steroid theory. Transl Psychiatry. 2018;8(1):136. https://doi.org/10.1038/ s41398-018-0186-7.

18. Crider A, Thakkar R, Ahmed AO, Pillai A. Dysregulation of estrogen receptor beta (ERbeta), aromatase (CYP19A1), and ER co-activators in the middle frontal gyrus of autism spectrum disorder subjects. Mol Autism. 2014;5(1):46. https://doi.org/10.1186/2040-2392-5-46.

19. Croen LA, Zerbo O, Qian Y, Massolo ML, Rich S, Sidney S, Kripke C. The health status of adults on the autism spectrum. Autism. 2015;19(7):81423. https://doi.org/10.1177/1362361315577517.

20. Davis EP, Pfaff D. Sexually dimorphic responses to early adversity: implications for affective problems and autism spectrum disorder. Psychoneuroendocrinology. 2014. https://doi.org/10.1016/j.psyneuen.2014.06.014

21. Gong S, Sovio U, Aye IL, Gaccioli F, Dopierala J, Johnson MD, Wood AM, et al. Placental polyamine metabolism differs by fetal sex, fetal growth restriction, and preeclampsia. JCI Insight. 2018. https://doi.org/10.1172/ jci.insight.120723.

22. Goodwin TM, Montoro M, Mestman JH, Eugene Pekary A, Hershman $\mathrm{JM}$. The role of chorionic gonadotropin in transient hyperthyroidism of hyperemesis gravidarum. J Clin Endocrinol Metab. 1992. https://doi.org/ 10.1210/jcem.75.5.1430095

23. Greenberg DM, Warrier V, Allison C, Baron-Cohen S. Testing the empathizing-systemizing theory of sex differences and the extreme male brain theory of autism in half a million people. Proc Natl Acad Sci USA. 2018. https://doi.org/10.1073/pnas.1811032115.

24. Harris PA, Taylor R, Minor BL, Elliott V, Fernandez M, O'Neal L, McLeod L, et al. The REDCap Consortium: building an international community of software platform partners. J Biomed Inform. 2019. https://doi.org/10. 1016/j.jbi.2019.103208.

25. Harris PA, Taylor R, Thielke R, Payne J, Gonzalez N, Conde JG. Research electronic data capture (REDCap) — A metadata-driven methodology and workflow process for providing translational research informatics support. J Biomed Inf. 2009. https://doi.org/10.1016/j.jbi.2008.08.010.

26. Hogeveen KN, Cousin P, Pugeat M, Dewailly D, Soudan B, Hammond GL. Human sex hormone-binding globulin variants associated with hyperandrogenism and ovarian dysfunction. J Clin Investig. 2002. https://doi.org/ 10.1172/JCl0214060.

27. Howlin P, Asgharian A. The diagnosis of autism and asperger syndrome: findings from a survey of 770 families. Dev Med Child Neurol. 1999. https://doi.org/10.1017/S0012162299001656.

28. Hu VW, Nguyen A, Kim KS, Steinberg ME, Sarachana T, Scully MA, Soldin SJ, LuU T, Lee NH. Gene expression profiling of lymphoblasts from autistic and nonaffected sib pairs: altered pathways in neuronal development and steroid biosynthesis. PLOS ONE. 2009;4(6): e5775. https://doi.org/10. 1371/journal.pone.0005775.

29. Knickmeyer R, Baron-Cohen S, Raggatt P, Taylor K. Foetal testosterone, social relationships, and restricted interests in children. J Child Psychol Psychiatry. 2005;46(2):198-210. https://doi.org/10.1111/j.1469-7610.2004. 00349.x.

30. Kosidou K, Dalman C, Widman L, Arver S, Lee BK, Magnusson C, Gardner RM. Maternal polycystic ovary syndrome and the risk of autism spectrum disorders in the offspring: a population-based nationwide study in Sweden. Mol Psychiatry. 2016;21(10):1441-8. https://doi.org/10.1038/mp 2015.183.

31. Lai MC, Lombardo MV, Suckling J, Ruigrok AN, Chakrabarti B, Ecker C, Deoni SC, et al. Biological sex affects the neurobiology of autism. Brain. 2013;136(Pt 9):2799-815. https://doi.org/10.1093/brain/awt216.

32. Lai M-C, Szatmari P. Sex and gender impacts on the behavioural presentation and recognition of autism. Curr Opin Psychiatry. 2020;33(2):117-23. https://doi.org/10.1097/YCO.0000000000000575.

33. Loomes R, Hull L, Mandy WPL. What is the male-to-female ratio in autism spectrum disorder? A systematic review and meta-analysis. J Am Acad Child Adolesc Psychiatry. 2017;56(6):466-74. https://doi.org/10.1016/j. jaac.2017.03.013.

34. Lutchmaya S, Baron-Cohen S, Raggatt P. Foetal testosterone and eye contact in 12-month-old human infants. Infant Behav Dev. 2002;25(3):32735. https://doi.org/10.1016/S0163-6383(02)00094-2.

35. Lutchmaya S, Baron-Cohen S, Raggatt P. Foetal testosterone and vocabulary size in 18- and 24-month-old infants. Infant Behav Dev. 2001. https:// doi.org/10.1016/S0163-6383(02)00087-5.

36. Maher GM, O'Keeffe GW, Kearney PM, Kenny LC, Dinan TG, Mattsson $M$, Khashan AS. Association of hypertensive disorders of pregnancy with risk of neurodevelopmental disorders in offspring. JAMA Psychiat. 2018;75(8):809. https://doi.org/10.1001/jamapsychiatry.2018.0854.

37. Maliqueo M, Lara HE, Sánchez F, Echiburú B, Crisosto N, Sir-Petermann T. Placental steroidogenesis in pregnant women with polycystic ovary 
syndrome. Eur J Obstet Gynecol Reprod Biol. 2013. https://doi.org/10. 1016/j.ejogrb.2012.10.015.

38. Murji A, Proctor LK, Paterson AD, Chitayat D, Weksberg R, Kingdom J. Male sex bias in placental dysfunction. Am J Med Genet A. 2012;158A(4):77983. https://doi.org/10.1002/ajmg.a.35250.

39. Pasquali R, Zanotti L, Fanelli F, Mezzullo M, Fazzini A, Labate AMM, Repaci A, Ribichini D, Gambineri A. Defining hyperandrogenism in women with polycystic ovary syndrome: a challenging perspective. J Clin Endocrinol Metab. 2016. https://doi.org/10.1210/jc.2015-4009.

40. Pohl A, Cassidy S, Auyeung B, Baron-Cohen S. Uncovering steroidopathy in women with autism: a latent class analysis. Mol Autism. 2014;5:27. https://doi.org/10.1186/2040-2392-5-27.

41. Roman-Urrestarazu A, Yáñez C, López-Garí C, Elgueta C, Allison C, Brayne C, Troncoso M, Baron-Cohen S. Autism screening and conditional cash transfers in Chile: using the quantitative checklist (Q-CHAT) for early autism detection in a low resource setting. Autism. 2020. https://doi.org/ $10.1177 / 1362361320972277$.

42. Ruta L, Ingudomnukul E, Taylor K, Chakrabarti B, Baron-Cohen S. Increased serum androstenedione in adults with autism spectrum conditions. Psychoneuroendocrinology. 2011;36(8):1154-63. https://doi.org/10. 1016/j.psyneuen.2011.02.007.

43. Ruta L, Chiarotti F, Arduino GM, Apicella F, Leonardi E, Maggio R, Carrozza C, et al. Validation of the quantitative checklist for autism in toddlers in an Italian clinical sample of young children with autism and other developmental disorders. Front Psychiatry. 2019;10:488. https://doi.org/10.3389/ fpsyt.2019.00488.

44. Sirmans SM, Pate KA. Epidemiology, diagnosis, and management of polycystic ovary syndrome. Clin Epidemiol. 2013;6(1):1-13. https://doi.org/10. 2147/clep.s37559.

45. Sollberger S, Ehlert U. How to use and interpret hormone ratios. Psychoneuroendocrinology. 2016;63(January):385-97. https://doi.org/10.1016/J. PSYNEUEN.2015.09.031.

46. Spencer K, Macri JN, Aitken DA, Connor JM. Free B-HCG as first-trimester marker for fetal trisomy. Lancet. 1992. https://doi.org/10.1016/01406736(92)92073-O.

47. Steier JA, Myking OL, Bergsjø PB. Correlation between fetal sex and human chorionic gonadotropin in peripheral maternal blood and amniotic fluid in second and third trimester normal pregnancies. Acta Obstet Gynecol Scand. 1999. https://doi.org/10.1080/j.1600-0412.1999. 780504.x.

48. Tan DW, Gilani SZ, Maybery MT, Mian A, Hunt A, Walters M, Whitehouse AJO. Hypermasculinised facial morphology in boys and girls with autism spectrum disorder and its association with symptomatology. Sci Rep. 2017;7(1):9348. https://doi.org/10.1038/s41598-017-09939-y.

49. Tan DW, Maybery MT, Gilani SZ, Alvares GA, Mian A, Suter D, Whitehouse AJO. A broad autism phenotype expressed in facial morphology. Transl Psychiatry. 2020. https://doi.org/10.1038/s41398-020-0695-z.

50. van de Beek C, Thijssen JH, Cohen-Kettenis PT, van Goozen SH, Buitelaar JK. Relationships between sex hormones assessed in amniotic fluid, and maternal and umbilical cord serum: what is the best source of information to investigate the effects of fetal hormonal exposure? Horm Behav. 2004;46(5):663-9. https://doi.org/10.1016/j.yhbeh.2004.06.010.

51. Welsh M, Suzuki H, Yamada G. The masculinization programming window. Endocr Dev. 2014;27:17-27. https://doi.org/10.1159/000363609.

52. Whitehouse AJO, Alvares GA, Cleary D, Harun A, Stojanoska A, Taylor LJ, Varcin KJ, Maybery M. Symptom severity in autism spectrum disorder is related to the frequency and severity of nausea and vomiting during pregnancy: a retrospective case-control study. Mol Autism. 2018;9(1):37. https://doi.org/10.1186/s13229-018-0223-7.

53. Windham GC, Lyall K, Anderson M, Kharrazi M. Autism spectrum disorder risk in relation to maternal mid-pregnancy serum hormone and protein markers from prenatal screening in California. J Autism Dev Disord. 2016;46(2):478-88. https://doi.org/10.1007/s10803-015-2587-2.

54. Wright D, Spencer K, Kagan K, Tørring N, Petersen OB, Christou A, Kallikas J, Nicolaides KH. First-trimester combined screening for trisomy 21 at 7-14 weeks' gestation. Ultrasound Obstet Gynecol. 2010. https://doi.org/ 10.1002/uog.7755.

\section{Publisher's Note}

Springer Nature remains neutral with regard to jurisdictional claims in published maps and institutional affiliations.
Ready to submit your research? Choose BMC and benefit from:

- fast, convenient online submission

- thorough peer review by experienced researchers in your field

- rapid publication on acceptance

- support for research data, including large and complex data types

- gold Open Access which fosters wider collaboration and increased citations

- maximum visibility for your research: over $100 \mathrm{M}$ website views per year

At BMC, research is always in progress.

Learn more biomedcentral.com/submissions 